\title{
Molecular mechanisms of hydrogen sulfide against uremic accelerated atherosclerosis through cPKC $\beta$ II/Akt signal pathway
}

\author{
Ruifang Xiong ${ }^{\dagger}$, Xiangxue Lu+, Jinghong Song, Han Li ${ }^{*}$ (D) and Shixiang Wang
}

\begin{abstract}
Background: Cardiovascular disease is the most common complication and leading cause of death in maintenance hemodialysis patients. The protection mechanism of hydrogen sulfide $\left(\mathrm{H}_{2} \mathrm{~S}\right)$ and the specific role of conventional protein kinase C $\beta \|$ (CPKC $\beta \|) / A k t$ signaling pathway in the formation of atherosclerosis is still controversial.

Methods: 8-week-old male ApoE ${ }^{-/-}$mice were treated with 5/6 nephrectomy and high-fat diet to make uremia accelerated atherosclerosis (UAAS) model. Mice were divided into normal control group (control group), sham operation group (sham group), UAAS group, L-cysteine group (UAAS+L-cys group), sodium hydrosulfide group (UAAS+NaHS group), and propargylglycine group (UAAS+PPG group). Western blot was used to detect cPKC $\beta$ II activation, Akt phosphorylation and endothelial nitric oxide synthase (eNOS) expression in mice aorta.

Results: The membrane translocation of $C P K C \beta \|$ in UAAS group was higher than sham group, and L-cys or NaHS injection could suppress the membrane translocation, but PPG treatment resulted in more membrane translocation of $\mathrm{CPKC} B \|$ ( $P<0.05, n=6$ per group). Akt phosphorylation and the eNOS expression in UAAS group was lower than sham group, and L-cys or NaHS injection could suppress the degradation of Akt phosphorylation and the eNOS expression, but PPG treatment resulted in more decrease in the Akt phosphorylation and the eNOS expression ( $P<0.05, n=6$ per group).

Conclusion: Endogenous cystathionine- $\gamma$-lyase $(\mathrm{CSE}) / \mathrm{H}_{2} \mathrm{~S}$ system protected against the formation of UAAS via CPKCBII/Akt signal pathway. The imbalance of $\mathrm{CSE} / \mathrm{H}_{2} \mathrm{~S}$ system may participate in the formation of UAAS by affecting the expression of downstream molecule eNOS, which may be mediated by CPKC $\beta$ II/Akt signaling pathway.
\end{abstract}

Keywords: Uremia accelerated atherosclerosis, $\mathrm{ApoE}^{-/-}$mice, Hydrogen sulfide, cPKCßII/Akt signaling pathway, Endothelial nitric oxide synthase

\section{Background}

Cardiovascular disease is the most common complication and leading cause of death in maintenance hemodialysis patients [1]. The incidence of cardiovascular disease in chronic kidney disease (CKD) patients is $80 \%$, and the risk of death from cardiovascular events in hemodialysis patients is 10 to 20 times that of the general population [2]. The episode age of atherosclerosis is advanced, and vascular lesions progress faster in patients

\footnotetext{
* Correspondence: hanli@ccmu.edu.cn

${ }^{+}$Ruifang Xiong and Xiangxue Lu contributed equally to this work. Department of Blood Purification, Beijing Chao-Yang Hospital, Capital Medical University, No. 8 Gongti South Road, Chaoyang District, Beijing 100020, China
}

with end-stage renal disease, which is known as uremia accelerated atherosclerosis (UAAS), the age of onset is generally 30 to 40 [3]. As atherosclerosis is the main predictor of death of cardiovascular disease in hemodialysis patients, studies on occurrence and progression of atherosclerosis in uremic patients are of great significance for the prevention and treatment of cardiovascular diseases [4].

The damage of arterial endothelium is recognized as initial factor for atherosclerosis and plays an important role in the occurrence and development of cardiovascular diseases [5]. Our group found that there was a relationship between endogenous cystathionine- $\gamma$-lyase/ 
hydrogen sulfide $\left(\mathrm{CSE} / \mathrm{H}_{2} \mathrm{~S}\right)$ system and the risk of cardiovascular disease in maintenance hemodialysis patients [6]. Abnormal metabolism of endogenous $\mathrm{H}_{2} \mathrm{~S}$ accelerated the progression of atherosclerosis in patients with diabetic nephropathy [7]. We further found that uremia accelerated the progression of atherosclerosis in $\mathrm{ApoE}^{-/-}$ mice and exogenous $\mathrm{H}_{2} \mathrm{~S}$ can inhibit the progression of atherosclerosis in uremia $\mathrm{ApoE}^{-/-}$mice. Protein kinase $\mathrm{C}$ (PKC) is an important signal transduction molecule in cells [8]. Akt is one of the most versatile kinases in the human kinome and is critical regulator of human physiology that controls diverse cellular functions [9]. It was shown that the level of Akt phosphorylation was changed during the development and progression of atherosclerosis [10]. We found that $\mathrm{H}_{2} \mathrm{~S}$ level in patients with maintenance hemodialysis was lower than that in the normal population, with increased cPKC $\beta I I$ activation and decreased Akt phosphorylation [6]. Other studies have shown that $\mathrm{cPKC} \beta \mathrm{II}$ inhibitors can slow down the progression of cardiovascular disease [11]. These studies suggest that abnormal $\mathrm{H}_{2} \mathrm{~S}$ metabolism was involved in the progression of uremia with cardiovascular disease and may be achieved through PKC signaling pathways. $\mathrm{H}_{2} \mathrm{~S}$ and nitric oxide (NO) have a synergistic effect on vasodilation. Exogenous $\mathrm{H}_{2} \mathrm{~S}$ can enhance the relaxation of $\mathrm{NO}$ on blood vessels [12]. The activation of cPKC $\beta \mathrm{II}$ is increased in aortic endothelial cells of diabetic patients, and PKC inhibitors improved insulin-mediated endothelial nitric oxide synthase (eNOS) activation in patients with diabetes mellitus, which suggested that $\mathrm{H}_{2} \mathrm{~S}$ can regulate the production of eNOS through a certain pathway and participate in the regulation of endothelial function [13].

Large numbers of studies have shown that $\mathrm{H}_{2} \mathrm{~S}$ has a protective role in the formation of atherosclerosis, [14, 15] but its protection mechanism is still not clear. cPKC $\beta I I / A k t$ signaling pathway is widely involved in the occurrence and development of cardiovascular diseases such as atherosclerosis, myocardial ischemia-reperfusion injury, and heart failure, but the specific role of cPKC $3 I$ IIAkt signaling pathway in the formation of atherosclerosis remains controversial. The aim of this study was to identify the possible molecular mechanism of the $\mathrm{CSE} / \mathrm{H}_{2} \mathrm{~S}$ system and $\mathrm{cPKC} \beta \mathrm{II} /$ Akt signaling pathway on atherosclerosis development in UAAS mice.

\section{Methods}

\section{Materials}

BCA protein quantification kit (Applygen), sodium hydrosulfide (Sigma-aldrich), L-cysteine (Sinopharm), propargylglycine (Aladdin), Anti-eNOS Rabbit Antibody (Santa Cruz), Anti-p-Akt Mouse Antibody (Santa Cruz), Anti-tAkt Mouse Antibody (Santa Cruz), Anti-cPKCßII Rabbit Antibody (Santa Cruz), HRP-labeled Goat Anti-Mouse
IgG (Applygen), HRP-labeled Goat Anti-Rabbit IgG (Applygen), $\beta$-actin, Mouse mAb (Applygen), Multifunctional enzyme immunoassay (Thermo), Gel imager (Bio-Rad), Membrane and cytosol protein extraction kit (Beyotime).

\section{Animals and UAAS model preparation}

Male $\mathrm{ApoE}^{-/-}$mice were purchased from Beijing Vital River Laboratory Animal Technology Co., Ltd. (License No. SCXK (Beijing) 2016-0006). Mice were reared in the clean level laboratory with temperature of $18-25^{\circ} \mathrm{C}$ and relative humidity of $35-50 \%$, sufficient oxygen was provided, 4 mice per cage. Our operations are in accordance with the NIH Guide for the Care and Use of Laboratory Animals. All procedures with animals in this study were approved by the Institutional Animal Care and Use Committee of Beijing Chao-Yang Hospital, Capital Medical University.

Mice were divided into control group, sham group, UAAS group, L-cysteine group (UAAS+L-cys group), sodium hydrosulfide group (UAAS+NaHS group), and propargylglycine group (UAAS+PPG group) $(n=6$ for each group). NaHS (a donor of $\mathrm{H}_{2} \mathrm{~S}$ ) and L-cys (a precursor of $\mathrm{H}_{2} \mathrm{~S}$ generation) were used as a source of $\mathrm{H}_{2} \mathrm{~S}$, and PPG is a selective inhibitor of CSE, which is an important $\mathrm{H}_{2} \mathrm{~S}$-synthesizing enzyme [16]. At 8 weeks of age, the mice were anesthetized with sodium pentobarbital by intraperitoneal injection $(0.06 \mathrm{~g} / \mathrm{kg})$. A longitudinal incision of approximately $1 \mathrm{~cm}$ was made at the lower lateral of the left costovertebral angle of the mice. Then the left kidney was exposed and the renal capsule was isolated. A total of $2 / 3$ of the kidney tissue was removed from the upper and lower poles of the left kidney, then a gelatin sponge was used to compress and stop bleeding. After that, the muscles and skin were sutured. After 2 weeks, the right kidney was exposed in the same way, and the right renal pedicle was ligated. After confirming complete ligation, the right kidney was excised [17]. In the sham group, the kidneys were only exposed during the two operations and no surgical resection was performed. All mice were fed a highfat diet (Cat. No.: D12108C). After the operation, L-cys(50 $\mathrm{mg} / \mathrm{kg} / \mathrm{d}), \quad \mathrm{NaHS}(56 \mu \mathrm{mol} / \mathrm{kg} / \mathrm{d})$ and $\mathrm{PPG}(37.5 \mathrm{mg} / \mathrm{kg} / \mathrm{d})$ were intraperitoneally injected in UAAS+L-cys group, $\mathrm{UAAS}+\mathrm{NaHS}$ group, and UAAS+PPG group respectively for 6 weeks. After the intraperitoneally injection for 6 weeks, mice were euthanized via cervical dislocation according to the American Veterinary Medical Association and aortas were dissected and separated from fatty tissue, rinsed with normal saline and stored in $\mathrm{a}-80^{\circ} \mathrm{C}$ freezer.

\section{Extraction of cytosolic and membrane proteins}

The membrane protein extraction reagent $\mathrm{A}$ was added to the aortic tissue debris (PMSF was added 2 min earlier, make the final PMSF concentration of $1 \mathrm{mM}$ ), 
homogenized, sonicated, vortex reconstituted, then centrifuged at $700 \mathrm{~g}$ for $10 \mathrm{~min}$ at $4{ }^{\circ} \mathrm{C}$, the supernatant was collected as cytoplasmic protein. The membrane protein extraction reagent $\mathrm{B}$ was added to the precipitate (PMSF was added 2 min earlier, make the final PMSF concentration of $1 \mathrm{mM}$ ), homogenized, sonicated, vortex reconstituted, and centrifuged at $14000 \mathrm{~g}$ for $5 \mathrm{~min}$ at $4{ }^{\circ} \mathrm{C}$, then the membrane protein was collected and stored at $-80^{\circ} \mathrm{C}$ for use.

\section{Extraction of total protein}

100uL of Pre-chilled RIPA lysis buffer was added to the cleaved aortic tissue, homogenized, sonicated, vortex reconstituted, then centrifuged at $12000 \mathrm{~g}$ for $10 \mathrm{~min}$ at $4{ }^{\circ} \mathrm{C}$, the supernatant was collected as total protein, stored at $-80^{\circ} \mathrm{C}$ for use.

\section{Western blot}

Protein levels of cPKC $\beta I$ of cytoplasmic and membrane protein, cPKC $\beta I I, A k t$, phosphorylated-Akt (p-Akt), eNOS of total protein in mice aorta were analyzed by Western blot. The concentration of cytoplasmic, membrane protein and tissue total protein were detected by BCA method. $20 \mu \mathrm{g}$ of each sample were electrophoresed on a $10 \%$ SDS-polyacrylamide gel. The protein was electrophoresed at a voltage of $80 \mathrm{~V}$, and was increased to $120 \mathrm{~V}$ when the protein runs to the separation gel. After completion of electrophoresis, the protein was transferred from SDS-PAGE to polyvinylidene fluoride film (PVDF) at $12 \mathrm{~V}$ for $100 \mathrm{~min}$. The PVDF membranes were blocked with $10 \%$ skim milk which was formulated in Tris-HCI buffered saline solution (TBST) for $60 \mathrm{~min}$, then the membrane was washed for 3 times with TBST for $10 \mathrm{~min}$ each. The membrane was probed with the following antibodies: $\beta$-actin (goat anti-mouse, 1:5000 dilution, Applygen), cPKC $\beta I I$ (goat anti-rabbit, 1:5000 dilution, Santa Cruz), p-Akt (goat anti-rabbit, 1:5000 dilution, Santa Cruz), t-Akt (goat anti-rabbit, 1:5000 dilution, Santa Cruz), eNOS (goat anti-rabbit, 1:5000 dilution, Santa Cruz). The membrane was incubated overnight at $4{ }^{\circ} \mathrm{C}$, after 3 times with TBST for $10 \mathrm{~min}$ each, membranes were incubated with HRP-labeled Goat Anti-Mouse IgG (1:5000 dilution, Applygen) or HRP-labeled Goat Anti-Rabbit IgG (1:5000 dilution, Applygen). The membranes were placed on a bleaching shaker for $1 \mathrm{~h}$ at room temperature. A, B color mixture (1:1) was dropped on membrane and immediately placed in a gel imager (Bio-Rad, USA) until the best band was appeared.

\section{Statistical analysis of data}

Western blot results were semi-quantitatively analyzed using Image Lab software, SPSS22.0 statistical software (SPSS for Windows, Version 22.0, SPSS, USA) was used to statistically analyze the experimental data. Results were expressed as mean \pm standard deviation $(\bar{x} \pm s)$. One-Way ANOVA was used between groups and LSD method was used each group. $P<0.05$ was considered statistical significance. GraphPad Prism 6.0 statistical plotting software was used to plot data graphs.

\section{Results}

$\mathrm{CSE} / \mathrm{H}_{2} \mathrm{~S}$ regulates activation of $\mathrm{CPKC} \beta$ Il against formation of UAAS in mouse aorta (Fig. 1)

Aortic cytosolic and membrane protein was extracted and membrane translocation of cPKC $\beta I I$ was detected. The membrane translocation of cPKC $\beta I I$ was of no significant difference between sham group and control group ( $P=0.345, n=6)$; The membrane translocation of cPKC $\beta$ II in UAAS group was higher than sham group $(P=0.015, \mathrm{n}=6)$, meanwhile, compared with UAAS group, L-cys $(P=0.010, \mathrm{n}=6)$ or NaHS $(P=0.012, \mathrm{n}=6)$ injection could suppress the membrane translocation, but PPG treatment $(P=0.031, \mathrm{n}=6)$ resulted in more membrane translocation of cPKC $\beta I I$ with differences of statistical significance.

\section{$\mathrm{CSE} / \mathrm{H}_{2} \mathrm{~S}$ system has no effect on total protein $\mathrm{CPKC} \beta \mathrm{II}$ expression in mouse aorta (Fig. 2)}

Total protein of mouse aorta was extracted and the expression of cPKC $\beta I$ was compared between groups, there were no significant difference in $\mathrm{CPKC} \beta \mathrm{II}$ expression between each group.

\section{CSE/ $\mathrm{H}_{2} \mathrm{~S}$ regulates Akt phosphorylation against UAAS formation in mouse aorta (Fig. 3)}

Total mouse aortic protein was extracted and Akt phosphorylation was detected. Akt phosphorylation was of no significant difference between sham group and control group $(P=0.362, n=6)$; Akt phosphorylation in UAAS group was lower than sham group $(P=0.000001$, $\mathrm{n}=6$ ), meanwhile, compared with UAAS group, L-cys $(P=0.000054, \mathrm{n}=6)$ or NaHS $(P=0.000010, \mathrm{n}=6)$ injection could suppress the degradation of Akt phosphorylation, but PPG treatment $(P=0.005836, n=6)$ resulted in more decrease in the Akt phosphorylation with differences of statistical significance.

\section{$\mathrm{CSE} / \mathrm{H}_{2} \mathrm{~S}$ system regulates eNOS expression in mouse aorta against UAAS formation in mice aorta (Fig. 4)}

Total mouse aortic protein was extracted and expression of eNOS protein was detected. Expression of eNOS was of no significant difference between sham group and control group $(P=0.345, n=6)$; The expression of eNOS in UAAS group was lower than sham group $(P=0.034$, $\mathrm{n}=6$ ), meanwhile, compared with UAAS group, L-cys $(P=0.028, \mathrm{n}=6)$ or NaHS $(P=0.012, \mathrm{n}=6)$ injection could suppress the degradation of eNOS expression, but 

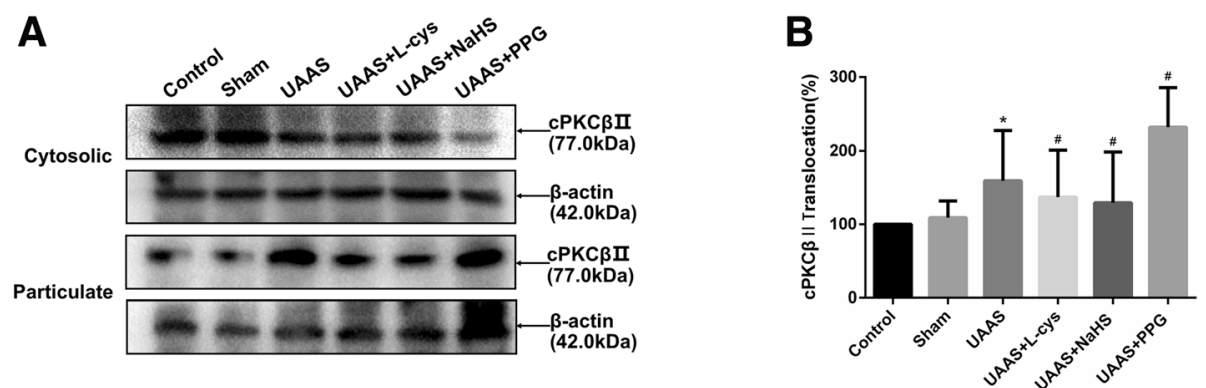

Fig. 1 Effects of CSE/ $\mathrm{H}_{2} \mathrm{~S}$ system on the membrane translocation of $\mathrm{CPKC} \beta \|$ in mouse aorta. The membrane translocation of $\mathrm{CPKC} \beta \|$ in control, sham, UAAS, UAAS+L-cys, UAAS+NaHS and UAAS+PPG group. (a) The protein contents in cytosolic and particulate fraction of mouse aorta were tested by Western blot; (b) Quantitative analysis showed that CPKC $\beta \|$ membrane translocation in UAAS group increased significantly compared with sham group $\left({ }^{*} P<0.05\right.$ vs. sham group, $n=6$ per group). L-cys or NaHS injection could suppress the membrane translocation, but PPG treatment resulted in more membrane translocation of $C$ PKC $\beta \| l\left({ }^{\#} P<0.05\right.$ vs. UAAS group, $n=6$ per group)

PPG treatment $(P=0.011, \mathrm{n}=6)$ resulted in more decrease in the eNOS expression with the difference of statistical significance.

\section{Discussion}

Patients with end-stage renal disease have increased cardiovascular morbidity and mortality [1]. Atherosclerosis is the main predictor of death of cardiovascular diseases in hemodialysis patients, and arterial endothelium damage is recognized as initial factor for atherosclerosis.

In mammals, $\mathrm{H}_{2} \mathrm{~S}$ is mainly produced through both enzymatic and nonenzymatic pathways. In enzyme synthesis pathway, endogenous $\mathrm{H}_{2} \mathrm{~S}$ is mainly produced by the metabolism of cysteine. Currently, there are five enzymes involved in the formation of $\mathrm{H}_{2} \mathrm{~S}$, namely cystathionine- $\beta$-synthase (CBS), cystathionine- $\gamma$-lyase (CSE), 3-mercaptopyruvate sulfurtransferase (3-MST), cysteine aminotransferase (CAT) and D- D-amino acid oxidase (DAO) [18]. Abnormal metabolism of endogenous $\mathrm{H}_{2} \mathrm{~S}$ accelerated the progression of atherosclerosis in patients with diabetic nephropathy [7]. Others showed that the occurrence of atherosclerosis and hypertension is associated with $\mathrm{CSE} / \mathrm{H}_{2} \mathrm{~S}$ disorders in chronic hemodialysis patients [19]. These findings indicate that endogenous $\mathrm{H}_{2} \mathrm{~S}$ has a certain inhibitory effect on the progression of atherosclerosis.

PKC is an important signal transduction molecule in cells. Currently PKC are divided into 3 subtypes, traditional PKC $(\alpha, \beta I, \beta I I, \gamma)$, novel PKC $(\delta, \varepsilon, \eta, \theta)$, atypical $\operatorname{PKC}(\zeta, \lambda)[8]$. Several studies have shown that cPKC $\beta I I$ plays very important role in the development of atherosclerosis, and its activation is the process of membrane translocation, that is, cPKC cytoplasm to the cell membrane [20].

Oxidized low density lipoprotein (oxLDL) activates cPKC 3 II through binding to LOX-1 receptor of human vascular endothelial cells, promotes NK activation, p66Shc, increases the production of reactive oxygen species, then accelerates the pathogenesis of atherosclerosis and the blocker of cPKC $\beta I I$ can inhibit the formation of atherosclerosis [21]. In diabetic $\mathrm{ApoE}^{-/-}$mice model, cPKC $\beta$ can damage vascular endothelial cells through
A

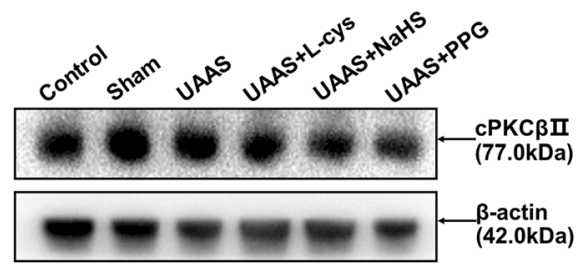

B

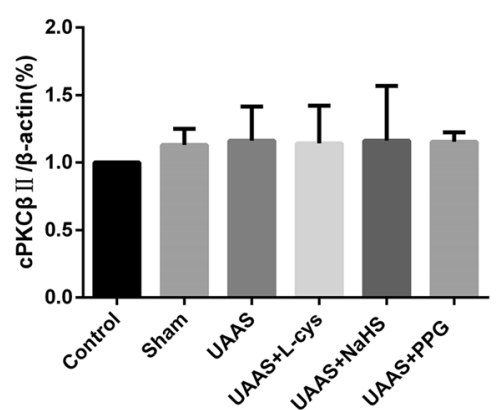

Fig. 2 Effects of $C S E / H_{2} S$ system on the total expression level of cPKC $\beta \|$ in mouse aorta. The total expression level of cPKC $\beta \|$ in control, sham, UAAS, UAAS+L-cys, UAAS+NaHS and UAAS+PPG group. (a) The protein contents in mouse aorta were tested by Western blot; (b) Quantitative analysis showed there were no significant difference in the expression of total cPKC $\beta \|$ in each group 

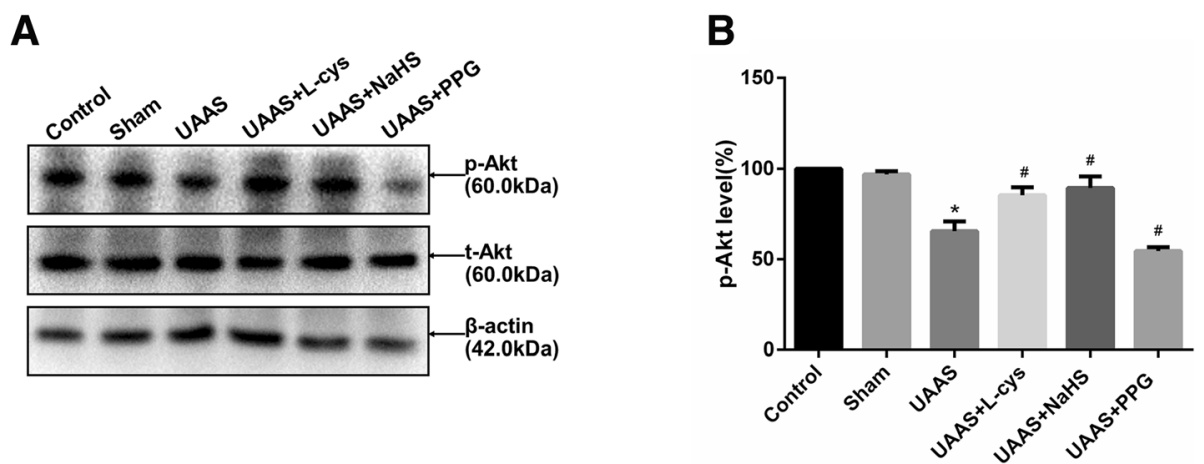

Fig. 3 Effects of $\mathrm{CSE} / \mathrm{H}_{2} \mathrm{~S}$ system on the phosphorylation level of Akt in mouse aorta. The phosphorylation level of Akt in control, sham, UAAS, UAAS+L-cys, UAAS+NaHS and UAAS+PPG group. (a) The protein contents in mouse aorta were tested by Western blot; (b) Quantitative analysis showed that Akt phosphorylation in UAAS group decreased significantly compared with sham group ( $P<0.05$ vs. sham group, $n=6$ per group). L-cys or NaHS injection could suppress the degradation of Akt phosphorylation, but PPG treatment resulted in more decrease in the Akt phosphorylation ( ${ }^{P} P<0.05$ vs. UAAS group, $n=6$ per group)

the IL-18/IL-18 binding protein pathway, promote the formation of atherosclerosis, and $\mathrm{cPKC} \beta$ inhibitor can relieve the progression of atherosclerosis [22]. Our group found that $\mathrm{H}_{2} \mathrm{~S}$ level in patients with maintenance hemodialysis was lower than that in the normal population, with increased cPKC $\beta$ II activation and decreased Akt phosphorylation [23]. Blocker of $\mathrm{cPKC} \beta \mathrm{II}$ in patients with cardiovascular disease may slow the progression of cardiovascular disease [11]. These studies suggest that abnormal $\mathrm{H}_{2} \mathrm{~S}$ metabolism was involved in the progression of uremia with cardiovascular disease and may be achieved through PKC signaling pathways.

$\mathrm{ApoE}^{-1-}$ mice act as one of the ideal animal models of hyperlipidemia and atherosclerosis [24]. Studies have shown that $\mathrm{ApoE}^{-1-}$ mice can develop atherosclerosis at 12 weeks on a high-fat diet [25]. Our previous study found that mice with $\mathrm{ApoE}^{-/-}$undergoing 5/6 nephrectomy and a high-fat diet for 6 weeks can successfully establish UAAS model. Aortic atherosclerotic plaques can be present in surgery group for 6 weeks. Exogenous $\mathrm{H}_{2} \mathrm{~S}$ donor NaHS delays formation of plaque formation in mice aorta to week 8 , plaque formation was observed 4 weeks after the administration of the CSE inhibitor PPG.

In this experiment, 8-week-old male $\mathrm{ApoE}^{-1-}$ mice were treated with 5/6 nephrectomy and high-fat diet to make UAAS model. Mice were divided into control group, sham group, UAAS group, UAAS+L-cys group, UAAS+NaHS group, and UAAS+PPG group. We found that the activation of cPKC $\beta I I$ was significantly increased in UAAS group compared with control group, with decreased phosphorylation of Akt. Compared with UAAS group, the activation of cPKC $\beta I I$ in UAAS+L-cys group and UAAS+NaHS group was decreased, with increased phosphorylation of Akt, suggesting that exogenous $\mathrm{H}_{2} \mathrm{~S}$ can inhibit membrane translocation of cPKC $\beta I I$, thus affect the activation of cPKCßII/Akt signaling pathway. In addition, compared with UAAS group, the activation of cPKC $\beta I$ II was significantly increased in UAAS+PPG
A

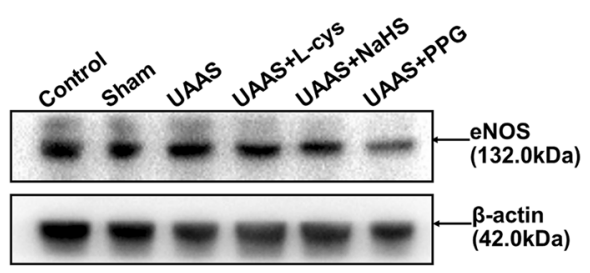

B

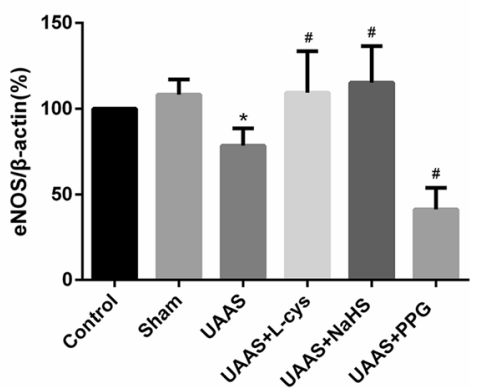

Fig. 4 Effects of CSE/ $H_{2} \mathrm{~S}$ system on the expression of eNOS in mouse aorta. The expression of eNOS in control, sham, UAAS, UAAS+L-Cys, UAAS+NaHS and UAAS+PPG group. (a) The protein contents in mouse aorta were tested by Western blot; (b) Quantitative analysis showed that eNOS expression in UAAS group decreased significantly compared with sham group ( $" P<0.05$ vs. sham group, $n=6$ per group). L-cys or NaHS injection could suppress the degradation of eNOS expression, but PPG treatment resulted in more decrease in the eNOS expression $(\# P<0.05$ vs. UAAS group, $n=6$ per group) 
group, with decreased phosphorylation of Akt, suggesting that exogenous CSE inhibitors can promote the membrane translocation of $\mathrm{cPKC} \beta \mathrm{II}$ and weaken the phosphorylation of Akt. The above results show that cPKC $3 \mathrm{II} /$ Akt signaling pathway is involved in the formation of accelerated atherosclerosis in uremia mice mediated by an imbalance of the $\mathrm{CSE} / \mathrm{H}_{2} \mathrm{~S}$ system.

$\mathrm{NO}$ is an important gas signaling molecule [26, 27]. There are three types of nitric oxide synthase known as neuronal nitric oxide synthase (nNOS), inducible nitric oxide synthase (iNOS), endothelial nitric oxide synthase (eNOS) [28]. The production of NO in endothelial cells mainly depends on eNOS. Studies have shown that NO can inhibit platelet adhesion, leukocyte accumulation, prevent proliferation of vascular smooth muscle cells, and also participate in the occurrence and development of coronary heart disease, heart failure, atherosclerosis, and thrombosis $[29,30]$. Decreased eNOS production or reduced biological activity can promote the formation of atherosclerosis by affecting the function of endothelial cells and the proliferation of smooth muscle cells [31].

$\mathrm{H}_{2} \mathrm{~S}$ and $\mathrm{NO}$ have a synergistic effect on vasodilation [32]. Exogenous $\mathrm{H}_{2} \mathrm{~S}$ can increase the relaxation of $\mathrm{NO}$ on blood vessels [12]. Experiments have shown that exogenous $\mathrm{H}_{2} \mathrm{~S}$ can alleviate the inhibitory effect of OXLDL on eNOS production, reduce oxidative stress and increase protection against vascular endothelium [33]. Exogenous $\mathrm{H}_{2} \mathrm{~S}$ can remit the inflammation of mice ear vein endothelial cells induced by phototoxicity by increasing the synthesis of eNOS, then inhibit the formation of intravascular thrombus [34]. These studies suggest that $\mathrm{H}_{2} \mathrm{~S}$ may regulate the production of eNOS through certain pathways, and thus affect the vascular endothelial function and participate in the formation of atherosclerosis. The activation of cPKC $\beta I I$ is increased in endothelial cells of patients with type 2 diabetes, and сPKC $\beta$ inhibitor LY379196 improved insulin-mediated eNOS activation. This process is involved in the occurrence of insulin resistance in diabetic patients [13]. Apigenin and naringenin can reduce the activation of cPKC $\beta I I$, up-regulate the expression of eNOS and inhibit the production of oxygen free radicals, then achieve the protective effect on endothelial cells [35]. It was also shown that cPKC $\beta I I$ could decrease eNOS activation in the wounded tissues of diabetic mice [36]. These studies suggest that $\mathrm{cPKC} \beta \mathrm{II}$ may participate in the regulation of vascular endothelial cell function by affecting the activity of eNOS in endothelial cells.

In this experimental study, the expression of eNOS was decreased in UAAS group compared with control group. Compared with UAAS group, the expression of eNOS was significantly increased in UAAS $+\mathrm{L}$-cys group and UAAS+NaHS group, the activation of cPKCßII was decreased, and the phosphorylation of Akt was increased. This suggests that the exogenous recruitment of $\mathrm{H}_{2} \mathrm{~S}$ donors and CSE substrates may affect the production of eNOS. In addition, compared with UAAS group, the expression of eNOS in UAAS+PPG group was decreased, the activation of membrane protein cPKC $\beta I I$ was increased, and the phosphorylation of Akt was decreased, suggesting that exogenous CSE synthetase inhibitors can inhibit the expression of eNOS. These results indicate that the imbalance of $\mathrm{CSE} / \mathrm{H}_{2} \mathrm{~S}$ system may influence the expression of downstream eNOS, regulate the production of $\mathrm{NO}$, achieve the effect on the vascular endothelial inflammation, and then regulate the formation of accelerated atherosclerosis in mice with uremia. This effect may be mediated by the cPKC $\beta I I /$ Akt signaling pathway. Our results of this experiment showed that the imbalance of $\mathrm{CSE} / \mathrm{H}_{2} \mathrm{~S}$ system in mice with accelerated uremic atherosclerosis was accompanied by activation of $\mathrm{cPKC} \beta \mathrm{II} /$ Akt signaling pathway and changes in eNOS. Suggesting that cPKC $\beta I /$ Akt signaling pathway may be involved in the regulation of downstream eNOS expression mediated by the unbalanced $\mathrm{CSE} / \mathrm{H}_{2} \mathrm{~S}$ system in mice with accelerated atherosclerosis. However, this experiment did not provide blocker of cPKC $\beta I I / A k t$ signaling pathway in mice, so we cannot exclude other signaling pathways may also participate in this process. And in addition to cPKC $\beta I I / A k t$ signaling pathway, are there any other pathways involved, or whether there are other downstream molecules working together, these require more in-depth research in vivo and in vitro experiments.

\section{Conclusion}

Endogenous $\mathrm{CSE} / \mathrm{H}_{2} \mathrm{~S}$ system may protect against the formation of UAAS via cPKCßII/Akt signal pathway. The imbalance of $\mathrm{CSE} / \mathrm{H}_{2} \mathrm{~S}$ system may participate in the formation of UAAS by affecting the expression of downstream molecule eNOS, which may be mediated by cPKC $\beta I I / A k t$ signaling pathway.

\section{Abbreviations \\ CKD: chronic kidney disease; CSE: cystathionine- $\gamma$-lyase; eNOS: endothelial nitric oxide synthase; $\mathrm{H}_{2} \mathrm{~S}$ : hydrogen sulfide; L-cys: L-cystein; NaHS: sodium hydrosulfide group; NO: nitric oxide; PKC: protein kinase $C_{\text {; }}$ \\ PPG: propargylglycine; PVDF: polyvinylidene fluoride film; UAAS: uremia accelerated atherosclerosis}

\section{Acknowledgments}

Not applicable.

\section{Authors' contributions}

$\mathrm{HL}$ and $\mathrm{S}-\mathrm{X} \mathrm{W}$ designed the experiment; R-F X and $X-X \mathrm{~L}$ wrote the paper; HL, S-X W, R-F X, X-X L and J-H S performed the experiments, collected and analyzed the data. All authors read and approved the final manuscript.

\section{Funding}

This study was funded by the National Natural Science Foundation of China (81670673) and Beijing Natural Science Foundation (7182060). Both of the funding bodies funded in the design of the study, collection, analysis, interpretation of data and writing the manuscript. 


\section{Availability of data and materials}

All the data supporting the conclusions of this article are contained within the manuscript.

\section{Ethics approval and consent to participate}

All procedures with animals in this study were approved by the Institutional Animal Care and Use Committee of Beijing Chao-Yang Hospital, Capital Medical University.

\section{Consent for publication}

Not applicable.

\section{Competing interests}

The authors declare that they have no competing interests.

Received: 8 May 2019 Accepted: 4 September 2019 Published online: 14 September 2019

\section{References}

1. Christoffersen C, Bartels ED, Aarup A, Nielsen LB, Pedersen TX. ApoB and apoM - new aspects of lipoprotein biology in uremia-induced atherosclerosis. Eur J Pharmacol. 2017;816:154-60.

2. Whitman IR, Feldman HI, Deo R. CKD and sudden cardiac death: epidemiology, mechanisms, and therapeutic approaches. J Am Soc Nephrol. 2012;23(12):1929-39.

3. Bosteen MH, Madsen Svarrer EM, Bisgaard LS, Martinussen T, Madsen M, Nielsen LB, Christoffersen C, Pedersen TX. Effects of apolipoprotein M in uremic atherosclerosis. Atherosclerosis. 2017;265:93-101.

4. Yamaguchi S, Gohda T, Gotoh H, Omote K, Furukawa M, Ishikawa Y, Tomino $Y$. Factors associated with cardiovascular death and events in patients with end stage renal disease. Nihon Jinzo Gakkai Shi. 2013;55(2):159-66.

5. Yamagata K. Docosahexaenoic acid regulates vascular endothelial cell function and prevents cardiovascular disease. Lipids Health Dis. 2017; 16(1):118

6. Feng SJ, Li H, Wang SX. Lower hydrogen sulfide is associated with cardiovascular mortality, which involves CPKCbetall/Akt pathway in chronic hemodialysis patients. Blood Purif. 2015:40(3):260-9.

7. Li H, Feng SJ, Zhang GZ, Wang SX. Correlation of lower concentrations of hydrogen sulfide with atherosclerosis in chronic hemodialysis patients with diabetic nephropathy. Blood Purif. 2014;38(3-4):188-94.

8. Li L, Xu S, Yan J, Li Y, Wang X, Du R, Zhu J, Fu X, Xiao X, Wang Z. Mechanism of PKC activity affecting the adhesion reaction of endothelial cells with monocytes. Int J Cardiol. 2015;182:361-7.

9. Gonzalez E, McGraw TE. The Akt kinases: isoform specificity in metabolism and cancer. Cell Cycle. 2009;8(16):2502-8.

10. Li Q, Park K, Xia Y, Matsumoto M, Qi W, Fu J, Yokomizo H, Khamaisi M, Wang $X$, Rask-Madsen C, et al. Regulation of macrophage apoptosis and atherosclerosis by lipid-induced PKCdelta isoform activation. Circ Res. 2017; 121(10):1153-67.

11. Wang G, Chen Z, Zhang F, Jing H, Xu W, Ning S, Li Z, Liu K, Yao J, Tian X. Blockade of PKCbeta protects against remote organ injury induced by intestinal ischemia and reperfusion via a p66shc-mediated mitochondrial apoptotic pathway. Apoptosis. 2014;19(9):1342-53.

12. Yetik-Anacak G, Sevin G, Ozzayim O, Dereli MV, Ahmed A. Hydrogen sulfide: a novel mechanism for the vascular protection by resveratrol under oxidative stress in mouse aorta. Vasc Pharmacol. 2016;87:76-82.

13. Tabit CE, Shenouda SM, Holbrook M, Fetterman JL, Kiani S, Frame AA, Kluge MA, Held A, Dohadwala MM, Gokce N, et al. Protein kinase C-beta contributes to impaired endothelial insulin signaling in humans with diabetes mellitus. Circulation. 2013;127(1):86-95.

14. Du C, Lin X, Xu W, Zheng F, Cai J, Yang J, Cui Q, Tang C, Cai J, Xu G, et al. Sulfhydrated sirtuin-1 increasing its deacetylation activity is an essential epigenetics mechanism of anti-atherogenesis by hydrogen sulfide. Antioxid Redox Signal. 2019;30(2):184-97.

15. Xu S, Liu Z, Liu P. Targeting hydrogen sulfide as a promising therapeutic strategy for atherosclerosis. Int J Cardiol. 2014;172(2):313-7.

16. Nalli AD, Bhattacharya S, Wang H, Kendig DM, Grider JR, Murthy KS. Augmentation of CGMP/PKG pathway and colonic motility by hydrogen sulfide. Am J Physiol Gastrointest Liver Physiol. 2017;313(4):G330-41.

17. Massy ZA, Ivanovski $O$, Nguyen-Khoa T, Angulo J, Szumilak D, Mothu N, Phan O, Daudon M, Lacour B, Drueke TB, et al. Uremia accelerates both atherosclerosis and arterial calcification in apolipoprotein E knockout mice. J Am Soc Nephrol. 2005;16(1):109-16.

18. Panthi S, Chung HJ, Jung J, Jeong NY. Physiological importance of hydrogen sulfide: emerging potent neuroprotector and neuromodulator. Oxidative Med Cell Longev. 2016;2016:9049782.

19. Weber GJ, Pushpakumar SB, Sen U. Hydrogen sulfide alleviates hypertensive kidney dysfunction through an epigenetic mechanism. Am J Physiol Heart Circ Physiol. 2017;312(5):H874-85.

20. Wuttke $A, Y u Q$, Tengholm A. Autocrine signaling underlies fast repetitive plasma membrane translocation of conventional and novel protein kinase $C$ isoforms in beta cells. J Biol Chem. 2016:291(29):14986-95.

21. Shi Y, Cosentino F, Camici GG, Akhmedov A, Vanhoutte PM, Tanner FC, Luscher TF. Oxidized low-density lipoprotein activates p66Shc via lectin-like oxidized low-density lipoprotein receptor-1, protein kinase C-beta, and cJun N-terminal kinase kinase in human endothelial cells. Arterioscler Thromb Vasc Biol. 2011;31(9):2090-7.

22. Durpes MC, Morin C, Paquin-Veillet J, Beland R, Pare M, Guimond MO, Rekhter M, King GL, Geraldes P. PKC-beta activation inhibits IL-18-binding protein causing endothelial dysfunction and diabetic atherosclerosis. Cardiovasc Res. 2015;106(2):303-13.

23. Wang W, Feng SJ, Li H, Zhang XD, Wang SX. Correlation of lower concentrations of hydrogen sulfide with activation of protein kinase Cbetall in uremic accelerated atherosclerosis patients. Chin Med J. 2015;128(11):1465-70.

24. Zhang Y, Li L, You J, Cao J, Fu X. Effect of 7-difluoromethyl-5, 4'dimethoxygenistein on aorta atherosclerosis in hyperlipidemia ApoE(-/-) mice induced by a cholesterol-rich diet. Drug Des Devel Ther. 2013:7:233-42.

25. Jawień J, Nastałek P, Korbut R. Mouse models of experimental atherosclerosis. J Physiol Pharmacol. 2004;55(3):503-17.

26. Fukuto JM, Carrington SJ, Tantillo DJ, Harrison JG, Ignarro LJ, Freeman BA, Chen A, Wink DA. Small molecule signaling agents: the integrated chemistry and biochemistry of nitrogen oxides, oxides of carbon, dioxygen, hydrogen sulfide, and their derived species. Chem Res Toxicol. 2012;25(4): 769-93.

27. Fix SM, Borden MA, Dayton PA. Therapeutic gas delivery via microbubbles and liposomes. J Control Release. 2015:209:139-49.

28. Oliveira-Paula GH, Lacchini R, Tanus-Santos JE. Inducible nitric oxide synthase as a possible target in hypertension. Curr Drug Targets. 2014;15(2): $164-74$

29. Zhao Y, Vanhoutte PM, Leung SW. Vascular nitric oxide: Beyond eNOS. J Pharmacol Sci. 2015;129(2):83-94.

30. Dusting GJ. Nitric oxide in coronary artery disease: roles in atherosclerosis, myocardial reperfusion and heart failure. EXS. 1996;76:33-55.

31. Tsikas D, Bollenbach A, Hanff E, Kayacelebi AA. Asymmetric dimethylarginine (ADMA), symmetric dimethylarginine (SDMA) and homoarginine (hArg): the ADMA, SDMA and hArg paradoxes. Cardiovasc Diabetol. 2018;17(1):1.

32. Kida M, Sugiyama T, Yoshimoto T, Ogawa Y. Hydrogen sulfide increases nitric oxide production with calcium-dependent activation of endothelial nitric oxide synthase in endothelial cells. Eur J Pharm Sci. 2013:48(1-2):211-5.

33. Lin Y, Chen Y, Zhu N, Zhao S, Fan J, Liu E. Hydrogen sulfide inhibits development of atherosclerosis through up-regulating protein Snitrosylation. Biomed Pharmacother. 2016;83:466-76.

34. Kram L, Grambow E, Mueller-Graf F, Sorg H, Vollmar B. The anti-thrombotic effect of hydrogen sulfide is partly mediated by an upregulation of nitric oxide synthases. Thromb Res. 2013;132(2):e112-7.

35. Qin W, Ren B, Wang S, Liang S, He B, Shi X, Wang L, Liang J, Wu F. Apigenin and naringenin ameliorate PKCbetall-associated endothelial dysfunction via regulating ROS/caspase-3 and NO pathway in endothelial cells exposed to high glucose. Vasc Pharmacol. 2016;85:39-49.

36. Das SK, Yuan YF, Li MQ. Specific PKC betall inhibitor: one stone two birds in the treatment of diabetic foot ulcers. Biosci Rep. 2018;38(5).

\section{Publisher's Note}

Springer Nature remains neutral with regard to jurisdictional claims in published maps and institutional affiliations. 